\title{
Intoxicação natural por Trema micrantha em caprinos
}

\author{
Spontaneosu poisoning by Trema micrantha in goats
}

\section{Sandra Davi Traverso ${ }^{1}$ Edson Moleta Colodel $^{2}$ Alexandre Paulino Loretti $^{3}$ André Mendes Correia ${ }^{4}$ David Driemeier ${ }^{5}$}

\section{RESUMO}

Dois caprinos confinados, que recebiam ração e pasto no cocho, morreram uma semana após serem alimentados com arbustos de plantas de diferentes espécies, entre os quais encontrava-se Trema micrantha. Apenas um animal foi necropsiado. Achados macroscópicos incluíram palidez generalizada da carcaça, hemorragias subcutâneas na região do esterno e escápula, figado amarelado, friável e com lobulação evidente. Na superficie de corte, o figado apresentou áreas vermelhas deprimidas entremeadas por áreas claras, deixando na faca, restos de conteúdo amarelado de aspecto gorduroso. Histologicamente, observouse necrose hepática centrolobular com hemorragia, associada à vacuolização de hepatócitos da região periportal. No encéfalo, havia vacuolização, degeneração e necrose de neurônios do córtex e hipocampo. Os indícios de ingestão da planta associado às lesões hepáticas descritas são compativeis com intoxicação por Trema micrantha.

Palavras-chave: intoxicação natural por planta Trema micrantha, Ulmaceae, caprinos.

\section{ABSTRACT}

Two goats which were housed and fed forages and commercial concentrate, died one week after being fed a mixture of plants. Amongst these plant was identified Trema micrantha. Only one animal was necropsied, and paleness of the carcass, subcutaneous haemorrhages in the region of chest and scapula, a yellowish and pliable liver with enhanced lobular pattern were the principal macroscopic findings. Additionally, in the cut surface from the liver, there were reddened and depressed areas mixed with withish ones. The most important histologic findings were hepatic centrilobular necrosis and haemorrhages associated with vacuolization of the periportal hepatocytes. In the encephalic tissue, there were vacuolisation, degeneration and necrosis of the neurones from cortex and hipocampus. The evidences of consumption of the plant and the hepatic lesions here described are consistent findings with the diagnosis of spontaneous poisoning by Trema micrantha.

Key words: spontaneous plant poisoning, Trema micrantha, Ulmaceae, goats.

\section{INTRODUÇÃO}

O gênero Trema (fam. Ulmaceae) inclui espécies de árvores de crescimento rápido, de surgimento natural após desmatamentos (CASTELLANI \& STUBBLEBINE, 1993). No Brasil, Trema micrantha tem sido utilizada nas recuperações de solos degradados ou após queimadas. (CASTELLANI \& AGUIAR, 1998). É uma planta

\footnotetext{
${ }^{1}$ Médico Veterinário, Mestrando Universidade Federal do Rio Grande do Sul (UFRGS), Faculdade de Veterinária (FAVET) - Setor de Patologia Veterinária. E-mail: kimtraverso@hotmail.com.

${ }^{2}$ Médico Veterinário, Professor da FUFMAT, Coxipó, 78060-900, Cuiabá, MT, Doutorando UFRGS-FAVET.

${ }^{3}$ Médico Veterinário, Mestre, Professor Assistente UFRGS-FAVET E-mail:aloretti@hotmail.com.

${ }^{4}$ Aluno do Curso de Graduação em Medicina Veterinária UFRGS-FAVET.

${ }^{5}$ Médico Veterinário, Bolsista CNPq. 301076/93-6, Professor Adjunto do Setor de Patologia Veterinária UFRGS, Av. Bento Gonçalves, 9090, CP 15094, 91540-000, Porto Alegre, RS. E-mail: davetpat@vortex.ufrgs.br. Autor para correspondência. 
perene, nativa do Brasil, arbórea, ereta, bastante ramificada, de caule estriado na cor marrom escura e que SE propaga somente por sementes (LORENZI, 2000).

As plantas do gênero Trema são palatáveis para animais de produção, embora seu maior consumo seja observado quando há escassez de alimento, por exemplo durante os meses de seca (MULHEARN, 1942). As derrubadas de árvores por ação de podas ou do vento constituem importantes fatores de risco nos casos de intoxicação por árvores, como é o caso das plantas do gênero Prunus sp. e Myoporum sp. (RIETCORREA et al., 1993) e Trema sp. No Estado de Santa Catarina, plantas do gênero Trema são cortadas e fornecidas no cocho para bovinos e há suspeita de intoxicação por Trema micrantha nesta mesma espécie Aldo Gava (Comunicação pessoal).

Trema micrantha foi descrita como tóxica experimentalmente em coelhos a partir de $35 \mathrm{~g} / \mathrm{kg}$. (TRAVERSO \& DRIEMEIER, 2000) e experimentalmente para cabras a partir de $30 \mathrm{~g} / \mathrm{kg}$ (TRAVERSO, 2002). Não há relatos prévios da intoxicação espontânea por esta espécie de planta em cabras. Intoxicações naturais por outra espécie de Trema, chamada de Trema tomentosa foram descritas em camelos (TRUEMAN \& POWELL, 1991), bovinos, caprinos, ovinos (MULHEARN, 1942), eqüinos (HILL et al., 1985) e veados (McKENZIE, 1985). Experimentalmente, T. tomentosa é tóxica para bovinos e caprinos nas doses de $2,29 \mathrm{~kg}$ e $344,25 \mathrm{~g}$, respectivamente (MULHEARN, 1942). As manifestações clínicas descritas nas diferentes espécies animais consistem de fraqueza, inapetência, salivação, tremores musculares e condição semi-comatosa nos estágios terminais (MULHEARN, 1942; McKENZIE et al. 1985 e TRUEMAN \& POWELL, 1991). Em eqüinos, os sinais clínicos eram semelhantes aos de cólica aguda, com postura rígida, relutância ao movimento, dispnéia, pulso rápido e com micções freqüentes (HILL et al., 1985). Achados macroscópicos observados em todos os caprinos e bovinos intoxicados experimentalmente por T. tomentosa consistem de hemorragias no tecido subcutâneo e serosas do pulmão e vísceras da cavidade abdominal. Em alguns casos, formando hematomas subserosos. O fígado apresenta consistência friável com lobulação evidente. (MULHEARN, 1942). As lesões histológicas descritas restringem-se ao fígado e consistem em necrose de coagulação, hemorragia acentuada e tumefação dos hepatócitos observada em camelos (TRUEMAN \& POWELL, 1991). Em coelhos intoxicados por Trema micrantha, lesões microscópicas adicionais àquelas encontradas no fígado, descritas acima, são observadas no encéfalo e cerebelo, sen- do caracterizadas por vacuolização, degeneração e necrose de neurônios do tronco cerebral, córtex, hipocampo, células de Purkinje e substância cinzenta da medula (TRAVERSO \& DRIEMEIER, 2000). O objetivo deste trabalho é relatar a intoxicação natural por Trema micrantha ocorrida em cabras no Estado do Rio Grande do Sul.

\section{MATERIAL E MÉTODOS}

Dados de manejo, alimentação e sinais clínicos foram obtidos com o proprietário dos animais. Necropsia e coleta de tecidos foram efetuados em um caprino durante a visita à propriedade. Amostras de tecidos foram processadas para exame histológico e coradas pela hematoxilina-eosina (HE).

\section{RESULTADOS}

Dois caprinos, que eram mantidos estabulados e recebiam ração e pasto no cocho, morreram uma semana após serem alimentados com arbustos de plantas de diferentes espécies, entre as quais encontrou-se Trema micrantha. A propriedade possuía 19 caprinos, dos quais, 17 eram mantidos soltos nas pastagens, e apenas os dois que morreram, eram confinados. Uma semana antes das mortes, uma área da propriedade onde havia árvores adultas, posteriormente identificadas como Trema micrantha, foi desmatada (Figura 1). Folhas verdes de T. micrantha foram cortadas após a derrubada das árvores e fornecidas aos dois animais mantidos estabulados. No animal necropsiado, observou-se discreta, mas generalizada icterícia da carcaça, com palidez generalizada da carcaça, hemorragias subcutâneas na região do esterno e escápula. O fígado estava amarelado, friável com padrão lobular muito evidente, e, ao corte, apresentava áreas vermelhas deprimidas entremeadas por áreas mais claras, deixando, na faca, resquícios de um líquido amarelado com aspecto de gordura (Figura 2). Edema de mesentério e vesícula biliar também foram observados. Na histologia, havia extensa necrose hepática centrolobular com áreas de hemorragia multifocal (Figura 3). Os hepatócitos da região periportal estavam vacuolizados. No encéfalo, havia vacuolização, degeneração e necrose de neurônios do córtex e do hipocampo. Musculatura do tronco e membros não apresentaram alterações histológicas.

\section{DISCUSSÃO}

As lesões da intoxicação por Trema micrantha são compatíveis com insuficiência hepáti- 


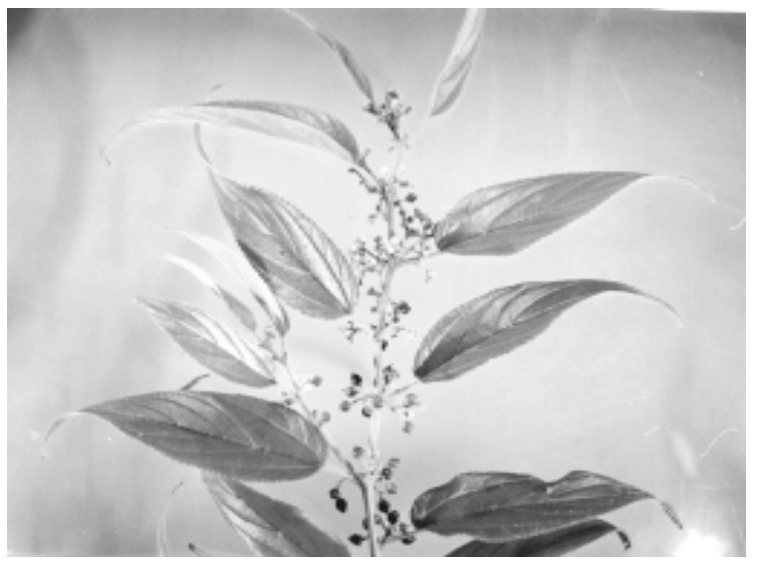

Figura 1 - Intoxicação espontânea por Trema micrantha em caprino. Ramo da árvore apresentando floração e frutificação. Novembro, 2000 .

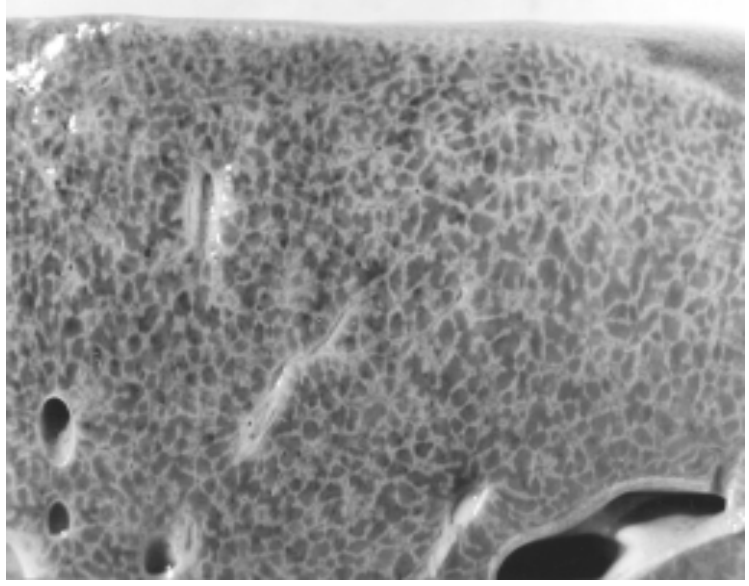

Figura 2 - Intoxicação espontânea por Trema micrantha em caprino. Fígado superfície de corte. Note o aspecto da lobulação evidenciada por linhas mais claras que correspondem histologicamente às áreas de vacuolização periportal.

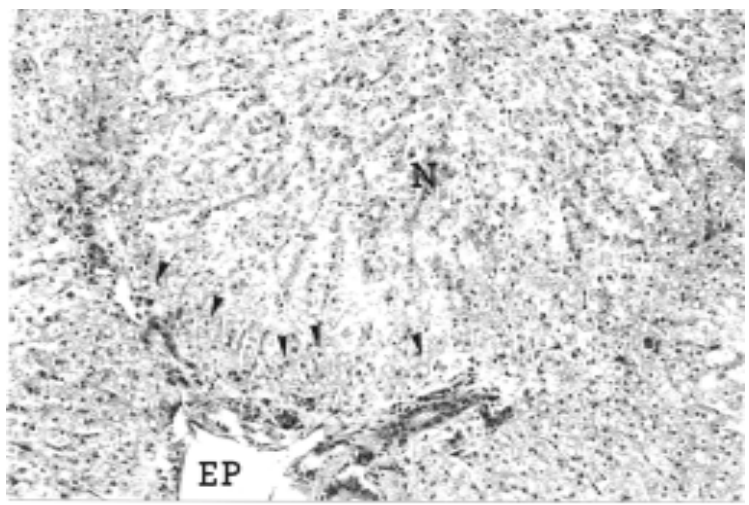

Figura 3 - Fígado - caprino. Intoxicação por Trema micrantha. Necrose (N) centrolobular acentuada e tumefação hepatocelular periportal (cabeça de setas). Espaço porta (EP). Hematoxilina-eosina, obj. 10X ca aguda, e para que isso ocorra, é necessário que $80 \%$ a $90 \%$ do parênquima esteja comprometido. Animais com insuficiência hepática morrem rapidamente, manifestando apatia, anorexia, diarréia, constipação e sinais nervosos caracterizados por hiperexcitabilidade, convulsão, coma, fraqueza, tremores musculares, andar compulsivo e pressão da cabeça contra a parede (KELLY, 1993). Muitos fatores, incluindo a hipoglicemia e a falência no mecanismo normal de detoxicação hepática, levam ao aumento excessivo de aminoácidos, amônia e produtos hepáticos tóxicos que podem ser a causa desses sintomas nervosos (RADOSTITS et al., 1994). Os sinais clínicos do caprino envolvido nesse relato não foram acompanhados pelos autores, entretanto as alterações microscópicas observadas no encéfalo indicam que sinais nervosos podem ter ocorrido.

A localização centrolobular, característica da necrose coagulativa hepatocelular, pode ser explicada pela distribuição das enzimas pertencentes ao sistema oxidase de função mista microssomal (MMFO), que estão presentes em maior quantidade nos hepatócitos dessa região (MACLACHLAN \& CULLEN, 1995). Tais enzimas são responsáveis pela transformação de compostos exógenos inativos em metabólitos tóxicos, o que explicaria a maior susceptibilidade dos hepatócitos da região centrolobular à agressão por metabólitos formados durante a detoxicação de diversos agentes químicos (KELLY, 1993). Adicionalmente, os hepatócitos da região centrolobular seriam particularmente mais vulneráveis a uma agressão tóxica, por estarem mais afastados da origem do sangue oxigenado do espaço porta (MACLACHLAN \& CULLEN, 1995).

Edema, hemorragias e alterações neurológicas são achados comuns na insuficiência hepática. $\mathrm{O}$ edema decorre da diminuição na formação de aminoácidos e proteínas, ocasionando queda nas proteínas plasmáticas e diminuição da pressão oncótica. As hemorragias observadas nesses casos devem-se à deficiência na formação de pró-trombina e, conseqüente, ao aumento no tempo de coagulação. Deficiência de fibrinogênio e tromboplastina também ocorrem e agravam as hemorragias (RADOSTITS et al., 1994). A alteração histológica no sistema nervoso central decorrente da grave lesão hepática que causa a hiperamonemia, é conhecida como degeneração esponjosa ou status espongiosus. Nesse caso, a lesão é caracterizada por vacuolização da substância branca (HOOPER, 1975). A hipoglicemia é um dos efeitos primários da lesão hepática aguda, e uma redução nos níveis sangüíneos de glicose pode ser responsável pelos sinais de encefalopatia hepática, em casos em que

Ciência Rural, v. 33, n. 1, jan-fev, 2003. 
não se verifica hiperamonemia. A lesão nervosa observada, neste caso, é caracterizada por cromatólise rápida e completo desaparecimento das margens citoplasmáticas, com posterior descoloração do citoplasma e picnose nuclear. As lesões histológicas do sistema nervoso central observadas na intoxicação por T. micrantha assemelham-se mais àquelas encontradas na hipoglicemia, que às encontradas na hiperamonemia. As lesões macro e microscópicas observadas no caprino são compatíveis com intoxicação por plantas hepatotóxicas. Na Região Sul, hepatopatias tóxicas agudas causadas por Xanthium cavanillesii (DRIEMEIER et al., 1999), Cestrum intermedium (GAVA et al.,1996), Cestrum corymbosum var. hirsutum (GAVA et al., 1991) e Cestrum parqui (RIET-CORREA et al., 1986) têm sido descritas e devem ser levadas em consideração no diagnóstico diferencial. O diagnóstico de intoxicação por Trema micrantha, neste estudo, está baseado na presença da planta no local, na ausência de outras plantas hepatotóxicas e na semelhança entre as lesões aqui observadas com aquelas encontradas na reprodução experimental em caprinos (TRAVERSO, 2002) e coelhos, induzida pela mesma planta (TRAVERSO \& DRIEMEIER, 2000). Os achados macroscópicos de hemorragias com palidez generalizada da carcaça associada à discreta icterícia observada no animal necropsiado eram bastante peculiares e causavam um aspecto de carne cozida a toda a musculatura. Estas mesmas alterações foram também constantemente observadas na reprodução experimental da lesão em cabras (TRAVERSO, 2002).

\section{AGRADECIMENTOS}

Os autores são gratos ao CNPq-Pronex pelo auxílio financeiro prestado ao Laboratório de Patologia Veterinária - Faculdade de Veterinária, UFRGS.

\section{REFERÊNCIAS BIBLIOGRÁFICAS}

CASTELLANI, T.T.; STUBBLEBINE WH. Early secondary succession after disturbance by fire in a semideciduous tropical forest. Rev Bras Botân, v.16, n.2, p.181-203, 1993.

CASTELLANI, E.D.; AGUIAR, I.B. Preliminary conditions for germination of Trema micrantha (L.) Blume seeds. Rev Bras Eng Agrícol Amb, v.2, n.1, p.80-83, 1998.

DRIEMEIER, D. et al. Intoxicação espontânea pelos frutos de Xanthium cavanilesii (Asteraceae) em bovinos no Rio Grande do Sul. Pesq Vet Bras, v.19, n.1, p.1-13, 1999.
GAVA, A. et al. Intoxicação por Cestrum curymbosum var. hirsutum (Solanaceae) em bovinos no Estado de Santa Catarina. Pes Vet Bras, v.11, n.3/4, p.71-74, 1991.

GAVA, A. et al. Intoxicação por Cestrum intermedium (Solanaceae) em bovinos. Pesq Vet Bras, v.16, n.4, p.117-120, 1996.

HILL, B.D.; WILLS, L.D.; DOWLING, R.M. Suspected poisoning of horses by Trema aspera (poison peach). Aust Vet J, v.62, n.3, p.107-108, 1985.

HOOPER, P.T. Spongy degeneration in the central nervous system of domestic animals. Part. III. Occurrence and pathogenesis hepatocerebral disease caused by hyperammonaemia. Acta Neuropath, v. 31, p.343-351, 1975.

KELLY, W.R. The liver and biliary system. In: JUBB, K.V.F, KENNEDY, P.C.; PALMER, N. Pathology of domestic animals. 4.ed San Diego : Academic, 1993. p.319-406.

LORENZI, H. Plantas daninhas do Brasil: terrestres aquáticas, parasitas e tóxicas. 3.ed. Nova Odessa : Plantarum, 2000. 623p.

MACLACHLAN, N.J.; CULLEN, J.M. Liver, biliary system, and exocrine pancreas. In: CARLTON, W.W.; MACGAVIN, M.D. Thomson's special pathology. 2.ed. Saint Louis : Mosby, 1995. p.81-115.

MCKENZIE, R.A. et al. Diseases of deer in south eastern Queensland. Aust Vet J, v.62, n.12, p.424, 1985.

MULHEARN, C.R. Poison peach (Trema aspera): a plant poisonous to stock. Aust Vet J, v.18, p.68-72, 1942.

RADOSTITS, O.M.; BLOOD, D.C.; GAY, C.C. Veterinary medicine. 8.ed. London : Baìllière Tindall, 1994. 1763 p.

RIET-CORREA, F. et al. Atividades do laboratório Regional de Diagnóstico e Doenças da Área de influência no período de 1978 a 1982. Pelotas : Universidade de Pelotas-RS, 1986. p.87-88. (Documento 4).

RIET-CORREA, F.; MÉNDEZ, M.C.; SCHILD, A. L. Intoxicações por plantas e micotoxicoses em animais domésticos. Pelotas: Hemisfério Sul, 1993. 340p.

TRAVERSO, S.D.; DRIEMEIER, D. Experimental Trema micrantha (Ulmaceae) poisoning in rabbits. Vet Hum Toxicol, v.42, n.5, p.301-302, 2000.

TRAVERSO S. D. Intoxicação experimental por Trema micrantha (Ulmaceae) em caprinos. .2002. 37f. Dissertação (Mestrado) - Programa de Pós-graduação em Ciências Veterinárias Universidade Federal do Rio Grande do Sul.

TRUEMAN, K.F.; POWELL, M.W. Suspected poisoning of camels by Trema tomentosa (poison peach). Aust Vet J, v.68, n.6, p.213214, 1991. 